\title{
Blind NLLS Carrier Frequency-Offset Estimation for QAM, PSK, and PAM Modulations: Performance at Low SNR
}

\author{
Philippe Ciblat and Mounir Ghogho
}

\begin{abstract}
We address the problem of blind carrier frequency-offset (CFO) estimation in quadrature amplitude modulation, phase-shift keying, and pulse amplitude modulation communications systems. We study the performance of a standard CFO estimate, which consists of first raising the received signal to the $M$ th power, where $M$ is an integer depending on the type and size of the symbol constellation, and then applying the nonlinear least squares (NLLS) estimation approach. At low signal-to noise ratio (SNR), the NLLS method fails to provide an accurate CFO estimate because of the presence of outliers. In this letter, we derive an approximate closed-form expression for the outlier probability. This enables us to predict the mean-square error (MSE) on CFO estimation for all SNR values. For a given SNR, the new results also give insight into the minimum number of samples required in the CFO estimation procedure, in order to ensure that the MSE on estimation is not significantly affected by the outliers.
\end{abstract}

Index Terms-Blind estimation, frequency-offset synchronization, outlier effect, performance at low signal-to-noise ratio (SNR), quadrature amplitude modulation (QAM) constellation.

\section{INTRODUCTION}

$\mathbf{I}$ $\mathrm{N}$ WIRELESS and wireline digital communications systems, the received signal may be corrupted by a carrier frequency-offset (CFO) due to Doppler shift and/or local oscillators drift. Since a CFO causes a time-varying rotation of the data symbols, it has to be accurately estimated and compensated for prior to symbol detection at the receiver, particularly in the case of large-size constellations. In this letter, we concentrate on non-data-aided (or blind) techniques in order to preserve bandwidth efficiency. Moreover, the considered blind frequency estimate is carrier-phase independent.

For the sake of simplicity, we consider linear modulation and a frequency-flat channel. The baud-sampled receive matched filter output can, after assuming perfect timing, be modeled as follows:

$$
y(n)=s(n) e^{2 i \pi\left(\phi_{0}+\phi_{1} n\right)}+b(n), \quad n=0, \cdots, N-1
$$

where $\{s(n)\}$ is a sequence of independent and identically distributed (i.i.d.) information-bearing symbols which are drawn from standard constellations such as quadrature amplitude modulation (QAM), phase-shift keying (PSK) or amplitude-shift keying (ASK), $b(n)$ is a circularly symmetric Gaussian white noise with variance $\sigma^{2}=\mathbb{E}\left[|b(n)|^{2}\right]$, and $N$

Paper approved by X. Dong, the Editor for Modulation and Signal Design of the IEEE Communications Society. Manuscript received May 20, 2005; revised February 21, 2006 and March 30, 2006.

P. Ciblat is with Département Communications et Electronique, Ecole Nationale Supérieure des Télécommunications (ENST), F-75013 Paris, France (e-mail: philippe.ciblat@enst.fr).

M. Ghogho is with the Department of Electronic and Electrical Engineering, University of Leeds, Leeds LS2 9JT, U.K. (e-mail: m.ghogho@leeds.ac.uk).

Digital Object Identifier 10.1109/TCOMM.2006.881341 is the number of available samples. Moreover, $\phi_{0}$ and $\phi_{1}$ are, respectively, the phase and CFO, which are unknown. More precisely, $\phi_{1}=2 \pi \Delta f T_{s}$, where $\Delta f$ is the frequency offset in Hertz and $T_{s}$ is the symbol period. To ensure the validity of (1), we assume that the effect of $\Delta f$ on the receive filter output can be neglected. Such an assumption is reasonable when $2 \pi \Delta f T_{s} \ll 1[1]$.

In the literature, several algorithms have been introduced to blindly estimate the frequency offset $\phi_{1}$ using the received signal sequence in (1) [2]-[7]. These methods are mainly based on the property that QAM, PSK, and pulse amplitude modulation (PAM) constellations obey a rotational symmetry of angle $2 \pi / M$, where $M$ is an integer proper to each set of constellations. For instance, $M=2$ for PAM, $M=4$ for QAM, and $M=P$ for $P$-PSK, with $P$ being the size of the PSK constellation [8]. This implies that $\mathbb{E}\left[s(n)^{M}\right] \neq 0$. Consequently, the signal $y(n)^{M}$ can be decomposed as

$$
y(n)^{M}=\mathcal{S} e^{2 i \pi M\left(\phi_{0}+\phi_{1} n\right)}+e(n)
$$

with

$$
\mathcal{S}=\mathbb{E}\left[s(n)^{M}\right]
$$

and

$$
\begin{aligned}
& e(n)=\left(s(n)^{M}-\mathcal{S}\right) e^{2 i \pi M\left(\phi_{0}+\phi_{1} n\right)} \\
& \quad+\sum_{m=0}^{M-1} C_{M}^{m} s(n)^{m} b(n)^{M-m} e^{2 i \pi m\left(\phi_{0}+\phi_{1} n\right)}
\end{aligned}
$$

and $C_{M}^{m}=(M ! / m !(M-m) !)$. The process $e(n)$, which can be considered as "noise," is a zero-mean white process with the following variance and pseudovariance:

$$
\begin{aligned}
\sigma_{e}^{2}= & \mathbb{E}\left[|e(n)|^{2}\right] \\
= & \left(\mathbb{E}\left[|s(n)|^{2 M}\right]-|\mathcal{S}|^{2}\right) \\
& +\sum_{m=0}^{M-1}\left(C_{M}^{m}\right)^{2} \mathbb{E}\left[|s(n)|^{2 m}\right] \mathbb{E}\left[|b(n)|^{2(M-m)}\right]
\end{aligned}
$$

and

$$
\tilde{\sigma}_{e}^{2}=\mathbb{E}\left[e(n)^{2}\right]=\left(\mathbb{E}\left[s(n)^{2 M}\right]-\mathcal{S}^{2}\right) e^{2 i \pi 2 M \phi_{0}} .
$$

Hence, the $M$ th power of the initial signal model in (1), which represents a complex exponential with frequency $\phi_{1}$ in multiplicative noise $s(n)$ and additive noise $b(n)$, can be seen as a constant-amplitude complex exponential with frequency $M \phi_{1}$ in a non-Gaussian but zero-mean additive noise $e(n)$ [2], [5], [7]. Therefore, the frequency $M \phi_{1}$ and phase $M \phi_{0}$ can be estimated using the nonlinear least squares (NLLS) approach, which ignores the statistical distribution of the additive noise. 
The NLLS estimate of $M \phi_{1}$ is obtained by maximizing the periodogram of $y(n)^{M}$ as follows [2], [5], [7]:

$$
\hat{\phi}_{1, N}=\frac{1}{M} \arg \max _{\phi \in(-1 / 2,1 / 2]}\left|\frac{1}{N} \sum_{n=0}^{N-1} y(n)^{M} e^{-2 i \pi \phi n}\right|^{2} .
$$

Although the cost function in (5) is not convex, the maximum is found by proceeding in two steps [9]:

- a coarse step which detects the maximum-magnitude peak which should be located around the frequency $M \phi_{1}$. This step may be carried out via a fast Fourier transform (FFT) of size $N(N-\mathrm{FFT})$;

- a fine step which inspects the cost function around the peak detected by the coarse step. This step may be implemented via a gradient-descent algorithm.

At low signal-to-noise ratio (SNR) and/or a small number of samples, the coarse step may detect a peak which is far away from the target point $M \phi_{1}$. In this case, the fine step becomes irrelevant, since performance is limited by the inaccurate peak detection in the coarse step. The failure of the coarse step is called the "outliers effect" [9]. As in [9], to simplify the analysis, let us assume that the sought frequency $\phi_{1}$ is the center of the search interval. Then, the true mean-square error (MSE) of the NLLS CFO estimate is

$$
\mathrm{MSE}=\frac{p}{12}+(1-p) \mathrm{MSE}_{\text {o.f. }}
$$

where $p$ is the probability of failure of the coarse step, also called the "outlier probability," and $\mathrm{MSE}_{\text {o.f. }}$ is the MSE when the outliers effect is not taken into account [9]. If the sought frequency $\phi_{1}$ is not the center of the search interval, a more complicated equation links the true MSE with the outlier probability; the factor $1 / 12$ should be replaced with another value which depends on the location of the sought frequency.

A closed-form expression for $\mathrm{MSE}_{\text {o.f. }}$ was derived in [4] and [7] for QAM, in [6] for PSK, and in [5] for PAM. However, no derivations for the outlier probability $p$ are available in the literature. The purpose of this letter is to fill this gap.

Previous works concerned with the derivation of the outlier probability only addressed the case where the additive noise is Gaussian and circularly symmetric [9]. In our case, the additive noise $e(n)$ is neither Gaussian nor circularly symmetric, in general. However, to make the derivation of the outlier probability analytically tractable, we impose a Gaussian distribution on $e(n)$. Since the outlier-free MSE (MSE o.f. $_{\text {) }}$ only depends on the variance and pseudovariance of $e(n)$ [4], [7], it is not affected by the Gaussian assumption. Thus, any mismatch between the empirical and proposed theoretical MSE, which assumes that $e(n)$ is Gaussian, is due to the outlier probability. Extensive simulations have shown that our theoretical MSE is always larger than the empirical MSE. It is, therefore, reasonable to conjecture that the Gaussian distribution of the noise is the worst distribution, as far as the MSE (including the outlier effect) of CFO estimation is concerned. However, since we do not have a formal proof for this result, we will refer to our theoretical MSE expressions as approximate expressions instead of upper bounds. Notice that even after imposing a Gaussian assumption on $e(n)$, the results presented in [9] cannot be applied to our problem, because $e(n)$ is not always circularly symmetric [cf. (4)]. Consequently, the expressions available in the literature cannot be used for our problem. The next section proposes closed-form expressions for the outlier probability when $e(n)$ is modeled by a Gaussian and probably noncircular white noise.

\section{OUtLIER PROBABILITy DeRIVATIONS}

In order to implement the coarse step, we compute the $N$-FFT of the sequence $\left\{y(n)^{M}, n=0, \ldots, N-1\right\}$ to obtain the frequency-domain sequence

$Y(k)=\frac{1}{N} \sum_{n=0}^{N-1} y(n)^{M} e^{-2 i \pi k n / N}, \quad k=-N / 2+1, \ldots, N / 2$

As mentioned in the previous section, we assume that the frequency $M \phi_{1}$ is null, and thus coincides with the central FFT frequency bin. Our theoretical analysis assumes that $N$ is even. The FFT algorithm further requires that $N$ is a power of two. Thus, using (2), we obtain

$$
|Y(k)|= \begin{cases}\left|\mathcal{S} e^{2 i \pi M \phi_{0}}+E(0)\right|, & \text { if } k=0 \\ |E(k)|, & \text { if } k \neq 0\end{cases}
$$

with

$$
E(k)=\frac{1}{N} \sum_{n=0}^{N-1} e(n) e^{-2 i \pi k n / N}
$$

and where $-N / 2+1 \leq k \leq N / 2$.

Before proceeding any further, we inspect the probability density function (pdf) of the random vector $\mathbf{E}=$ $[E(-N / 2+1), \cdots, E(N / 2)]$. After imposing the Gaussian assumption, $e(n)$ become an i.i.d. Gaussian process, and $\mathbf{E}$ is modeled by a Gaussian vector. Hence, the zero-mean vector $\mathbf{E}$ is completely characterized by its second-order statistics, i.e., its correlation function $\sigma_{E}^{2}\left(k, k^{\prime}\right)=\mathbb{E}\left[E(k) \overline{E\left(k^{\prime}\right)}\right]$, where the overline denotes the complex conjugate operator, and its pseudocorrelation (or conjugate-correlation) function $\tilde{\sigma}_{E}^{2}\left(k, k^{\prime}\right)=\mathbb{E}\left[E(k) E\left(k^{\prime}\right)\right]$. Straightforward algebraic manipulations lead to

$$
\sigma_{E}^{2}\left(k, k^{\prime}\right)=\frac{\sigma_{e}^{2}}{N} \delta_{k, k^{\prime}} \quad \text { and } \quad \tilde{\sigma}_{E}^{2}\left(k, k^{\prime}\right)=\frac{\tilde{\sigma}_{e}^{2}}{N} \delta_{k+k^{\prime}, 0} .
$$

As already mentioned in Section I, an outlier occurs when the coarse step fails, i.e., the maximum-magnitude peak of $Y(k)$ is not located at $k=0$. Since $E(k)$, for $k \neq 0$, is independent of $E(0)$ [cf. (6)], the outlier probability $p$ can be written as follows:

$$
\begin{aligned}
p & =1-\operatorname{Prob}(|Y(k)|<|Y(0)| \quad \forall k \neq 0) \\
& =1-\int_{0}^{+\infty} p_{1}(x) p_{2}(x) \mathrm{d} x
\end{aligned}
$$

where $p_{1}(x)=\operatorname{Prob}(|Y(k)|<x \quad \forall k \neq 0|| Y(0) \mid=x)$ and $p_{2}(x)=p_{|Y(0)|}(x)$ is the pdf of the random variable $|Y(0)|{ }^{1}$

\footnotetext{
${ }^{1}$ We use the same notation for both the random variable and its realization.
} 
Since $|E(k)|$ and $\left|E\left(k^{\prime}\right)\right|$ are independent when $k \neq \pm k^{\prime}$, we have that

$$
\begin{aligned}
p_{1}(x) & =\operatorname{Prob}(|E(N / 2)|<x|| Y(0) \mid=x) \\
& \times \prod_{k=1}^{N / 2-1} \operatorname{Prob}(|E(k)|<x,|E(-k)|<x|| Y(0) \mid=x) .
\end{aligned}
$$

Moreover, using the fact that the vectors $[|E(k)|,|E(-k)|]$, for $k=1 \ldots N / 2-1$, are i.i.d., we get

$$
p_{1}(x)=q_{1}(x)\left[q_{2}(x)\right]^{N / 2-1}
$$

with $q_{1}(x)=\operatorname{Prob}(|E(N / 2)|<x|| Y(0) \mid=x)$ and $q_{2}(x)=$ $\operatorname{Prob}(|E(k)|<x,|E(-k)|<x|| Y(0) \mid=x)$ where $k$ is any integer in $[1, N / 2-1]$ since $q_{2}(x)$ is independent of $k$. We next derive expressions for $q_{1}(x), q_{2}(x)$, and $p_{2}(x)$.

Derivation of $q_{1}(x)$ : The term $q_{1}(x)$ represents the probability that the modulus of a noncircularly symmetric complexvalued Gaussian variable is less than $x$. To derive $q_{1}(x)$, we thus need a closed-form expression for the pdf of $|E(N / 2)|$, which we denote by $p_{|E(N / 2)|}(\rho)$. Let $E_{R}=\Re\{E(N / 2)\}$ and $E_{I}=\Im\{E(N / 2)\}$, where $\Re\{$.$\} and \Im\{$.$\} stand for the real$ and imaginary parts of a complex-valued variable, respectively. We notice that the bivariate variable $\left[E_{R}, E_{I}\right]$ is a real-valued Gaussian vector with zero mean, and covariance matrix $\Sigma$ defined and given as follows:

$$
\begin{aligned}
\Sigma & :=\left[\begin{array}{cc}
\mathbb{E}\left[E_{R} E_{R}\right] & \mathbb{E}\left[E_{R} E_{I}\right] \\
\mathbb{E}\left[E_{I} E_{R}\right] & \mathbb{E}\left[E_{I} E_{I}\right]
\end{array}\right] \\
& =\frac{1}{2}\left[\begin{array}{cc}
\sigma_{E}^{2}+\Re\left(\tilde{\sigma}_{E}^{2}\right) & \Im\left(\tilde{\sigma}_{E}^{2}\right) \\
\Im\left(\tilde{\sigma}_{E}^{2}\right) & \sigma_{E}^{2}-\Re\left(\tilde{\sigma}_{E}^{2}\right)
\end{array}\right] .
\end{aligned}
$$

Consequently, the pdf of $\left[E_{R}, E_{I}\right]$, denoted by $p_{E_{R}, E_{I}}\left(e_{r}, e_{i}\right)$, takes the following form:

$$
p_{E_{R}, E_{I}}\left(e_{r}, e_{i}\right)=\frac{1}{2 \pi \sqrt{\operatorname{det}(\Sigma)}} e^{-\frac{1}{2}\left[e_{r}, e_{i}\right] \Sigma^{-1}\left[e_{r}, e_{i}\right]^{\mathrm{T}}}
$$

where the superscript ${ }^{\mathrm{T}}$ denotes transposition.

Let $R$ and $\Phi$ denote the modulus and angle of $|E(N / 2)|$, respectively. The pdf of the bivariate variable $[R, \Phi]$ can be expressed in terms of $p_{E_{R}, E_{I}}\left(e_{r}, e_{i}\right)$ as follows:

$$
p_{R, \Phi}(\rho, \varphi)=\rho p_{E_{R}, E_{I}}(\rho \cos (\varphi), \rho \sin (\varphi)) .
$$

This implies that

$$
\begin{aligned}
p_{|E(N / 2)|}(\rho):= & \int_{-\pi}^{\pi} p_{R, \Phi}(\rho, \varphi) \mathrm{d} \varphi \\
= & \frac{\rho}{2 \pi \sqrt{\operatorname{det}(\Sigma)}} \\
& \times \int_{-\pi}^{\pi} e^{-\frac{1}{2} \rho^{2}[\cos (\varphi), \sin (\varphi)] \Sigma^{-1}[\cos (\varphi), \sin (\varphi)]^{\mathrm{T}}} \mathrm{d} \varphi .
\end{aligned}
$$

Plugging (8) into the previous equation, and using the fact that $q_{1}(x)=\int_{0}^{x} p_{|E(N / 2)|}(\rho) \mathrm{d} \rho$ yield the following expression for $q_{1}(x)$ :

$$
q_{1}(x)=\frac{1}{\sqrt{u} \sigma_{e}^{2}} R\left(|r| ; u \sigma_{e}^{2} N x^{2}\right)
$$

with

$$
R(a ; y)=\int_{0}^{y} e^{-z} I_{0}(a z) \mathrm{d} z, \quad r=\frac{\tilde{\sigma}_{e}^{2}}{\sigma_{e}^{2}}, \quad u=\frac{1}{\left(\sigma_{e}^{4}-\left|\tilde{\sigma}_{e}^{2}\right|^{2}\right)}
$$

and $I_{0}(z)$ stands for the modified Bessel function of first kind.

Derivation of $q_{2}(x)$ : By following the same approach as above, we obtain

$q_{2}(x)=\int_{0}^{x} \int_{0}^{x} 4 \rho_{1} \rho_{2} u N^{2} e^{-u \sigma_{e}^{2} N\left(\rho_{1}^{2}+\rho_{2}^{2}\right)}$

$\times I_{0}\left(2 u\left|\tilde{\sigma}_{e}^{2}\right| N \rho_{1} \rho_{2}\right) \mathrm{d} \rho_{1} \mathrm{~d} \rho_{2}$.

Setting $\Omega_{1}=\Omega_{2}=\sigma_{e}^{2} / N$ and $\sqrt{\rho}=\left|\tilde{\sigma}_{e}^{2}\right| / \sigma_{e}^{2}$ in [10, eq. (6)], we get the following simplified expression:

$$
\begin{aligned}
q_{2}(x)=\left(1-e^{-N \frac{x^{2}}{\sigma_{e}^{2}}}\right)+ & e^{-N \frac{x^{2}}{\sigma_{e}^{2}}}\left[Q\left(\sqrt{2 u N} \frac{\left|\tilde{\sigma}_{e}^{2}\right|}{\sigma_{e}} x, \sqrt{2 u N} \sigma_{e} x\right)\right. \\
& \left.-Q\left(\sqrt{2 u N} \sigma_{e} x, \sqrt{2 u N} \frac{\left|\tilde{\sigma}_{e}^{2}\right|}{\sigma_{e}} x\right)\right]
\end{aligned}
$$

where $Q(\alpha, \beta)$ is the Marcum function, defined as $Q(\alpha, \beta)=$ $\int_{\beta}^{\infty} z e^{-\left(z^{2}+\alpha^{2}\right) / 2} I_{0}(\alpha z) \mathrm{d} z$.

Derivation of $p_{2}(x)$ : Again, by proceeding in a similar way as above, we find that $p_{2}(x)$ can be expressed as follows:

$$
\begin{aligned}
p_{2}(x)=2 \sqrt{u} & N x e^{-\left(u \sigma_{e}^{2} N x^{2}+u w N\right)} \\
& \times P\left(\tilde{\sigma}_{e}^{2} x^{2} / N, 2 \bar{v}\left(\tilde{\sigma}_{e}^{2}-\sigma_{e}^{2}\right) x / N ; N^{2} u\right)
\end{aligned}
$$

with

$$
\begin{aligned}
P(a, b ; z) & =\frac{1}{2 \pi} \int_{-\pi}^{\pi} e^{z \Re\left[a e^{-2 i \theta}-b e^{i \theta}\right]} \mathrm{d} \theta \\
v & =\mathcal{S} e^{2 i \pi M \phi_{0}} \\
w & =|v|^{2} \sigma_{e}^{2}-\Re\left[\tilde{\sigma}_{e}^{2} \bar{v}^{2}\right] .
\end{aligned}
$$

Final Result: By merging (9), (10), and (11), and by applying the change of variable $y^{2}=u \sigma_{e}^{2} N x^{2}$, we obtain the following final expression for the outlier probability:

$$
\begin{aligned}
p= & 1-\frac{2 e^{-u w N}}{u \sigma_{e}^{4}} \\
& \times \int_{0}^{\infty}\left[\left(1-e^{-\frac{y^{2}}{u \sigma_{e}^{4}}}\right)+e^{-\frac{y^{2}}{u \sigma_{e}^{4}}}[Q(\sqrt{2} r y, \sqrt{2} y)\right. \\
& \quad-Q(\sqrt{2} y, \sqrt{2} r y)]]^{N / 2-1} \\
& \times y e^{-y^{2}} R\left(|r|, y^{2}\right) \quad \\
& \times P\left(r y^{2} / u N^{2}, 2 \bar{v}\left(\tilde{\sigma}_{e}^{2}-\sigma_{e}^{2}\right) y /\left(\left|\sigma_{e}\right| \sqrt{u N} N\right) ; N^{2} u\right) \mathrm{d} y .
\end{aligned}
$$



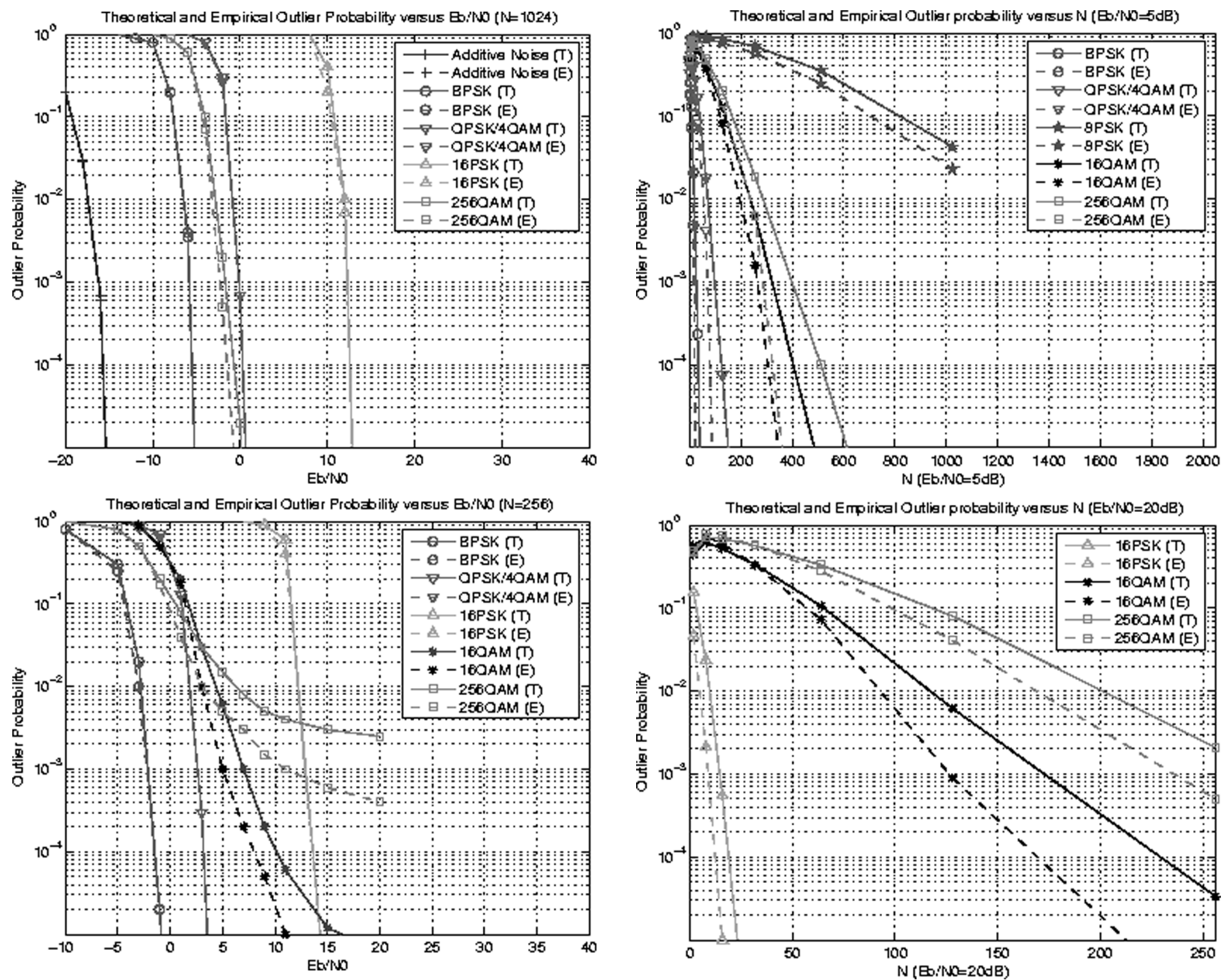

Fig. 1. Theoretical and empirical outlier probability versus $E_{b} / N_{0}$ (top: $N=$ 1024; bottom: $N=256$ ).

The following remarks are in order.

- In the case of data-aided CFO estimation, the $s(n)$ 's are known to the receiver and are often chosen to have constant magnitude. In this case, the received signal is first demodulated using $\tilde{y}(n):=y(n) / s(n)$, and then CFO estimation is carried out as in (5) after replacing $y(n)$ by $\tilde{y}(n)$ and setting $M=1$. Since the $E(k)$ 's are i.i.d. and circularly symmetric in this case, the outlier probability is obtained by setting $\tilde{\sigma}_{e}^{2}=0$ in the proposed expression given in (12) with $M=1$. In this case, our expression reduces to [9, eq. (59)] by setting $b_{0}=|\mathcal{S}|$ and $\sigma^{2}=\sigma_{e}^{2} / 2$.

- In the case of PSK constellations, the $E(k)$ 's are still i.i.d. and circularly symmetric. Therefore, the outlier probability in (12) can be simplified, since $\tilde{\sigma}_{e}^{2}=0$. Again, the obtained expression is equal to that in [9] when applied to the equivalent additive noise model in (2).

- In the case of QAM constellations, $E(k)$ 's are neither i.i.d. nor circularly symmetric. Therefore, $\tilde{\sigma}_{e}^{2} \neq 0$ and the expression in [9] does not apply anymore. Our expression for the outlier probability in (12) is an extension of that given

Fig. 2. Theoretical and empirical outlier probability versus $N$ (top: $E_{b} / N_{0}=$ $5 \mathrm{~dB}$; bottom: $E_{b} / N_{0}=20 \mathrm{~dB}$ ).

in [9] to include the case where the $E(k)$ 's are not circularly symmetric.

\section{NUMERICAL ILLUSTRATIONS}

In Fig. 1, the theoretical and empirical outlier probabilities are displayed versus the SNR $E_{b} / N_{0}\left(E_{b} / N_{0}=\right.$ $\mathbb{E}\left[|s(n)|^{2}\right] /\left(\log _{2}(P) \mathbb{E}\left[|b(n)|^{2}\right]\right)$, where $P$ is the size of the constellation) for different QAM and PSK constellations, as well as for the case of data-aided CFO estimation (i.e., the $s(n)$ 's are known and have the same magnitude denoted by $|s|)$. For the latter, the expression for the outlier probability derived in [9] is used here as a benchmark, and is referred to as "additive noise" in the legends of the figures; notice that since the transmit signal does not carry information in this case, the SNR cannot be defined as above. In this case, we use SNR $=|s|^{2} / \mathbb{E}\left[|b(n)|^{2}\right]$. We set $\phi_{0}=\phi_{1}=0$. The empirical outlier probability is obtained using 10000 Monte Carlo trials. We remark that the empirical and theoretical curves are in good agreement in the case of PSK constellations at all SNR values, and in the case of QAM constellations at low SNR. At high 

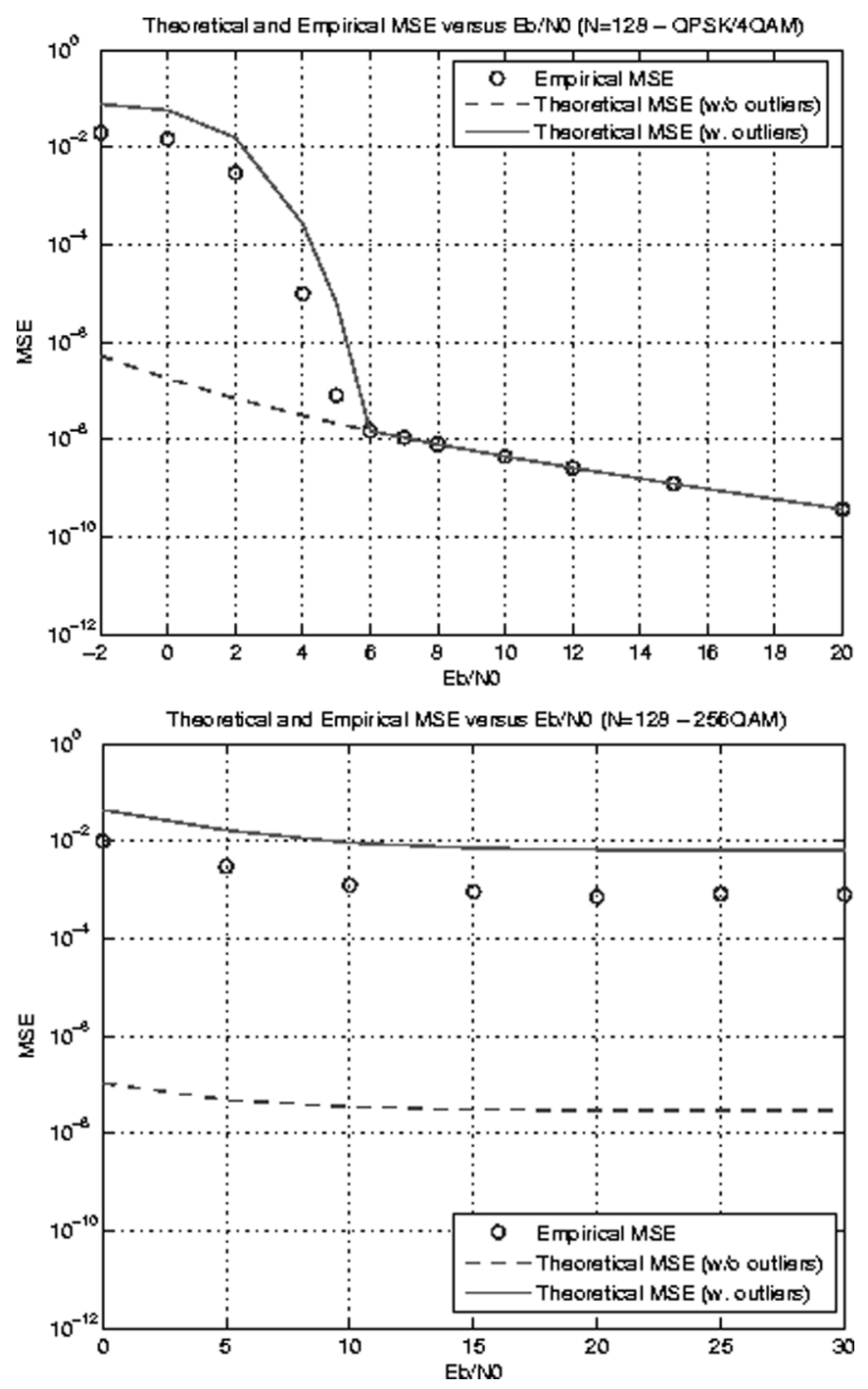

Fig. 3. Theoretical and empirical MSE versus $E_{b} / N_{0}$ (top: QPSK; bottom: 256QAM).

SNR, we observe that a floor effect occurs in the case of QAM with constellation size strictly larger than four. The theoretical prediction in this case is relatively too pessimistic (due to the Gaussian assumption on $e(n)$ ). The floor effect is caused by the self-noise induced by QAM constellations, i.e., $\sigma_{e}^{2}$ and $\tilde{\sigma}_{e}^{2}$ are nonzero, even in the absence of additive noise. Fig. 2 represents the outlier probabilities versus $N$. We remark that the theoretical and empirical curves are close to each other. We also observe that the outlier probability is slightly affected by the size of QAM constellations. This is mainly due to the fact that for QAM, $M=4$ regardless of the size of the constellation. In the case of PSK constellations, $M$ is equal to the constellation size; hence, performance dramatically degrades when $M$ increases. However, unlike QAM, PSK constellations do not suffer from the floor effect. Therefore, for a fixed $M$-PSK, one can rapidly reach a small value for the outlier probability by slightly increasing the SNR. Figs. 3 and 4 depict the MSE of the CFO estimate versus $E_{b} / N_{0}$ and $N$, respectively. The number of Monte-Carlo trials was set to 1000000 . To obtain the MSE that takes into account the outlier effect, we use the
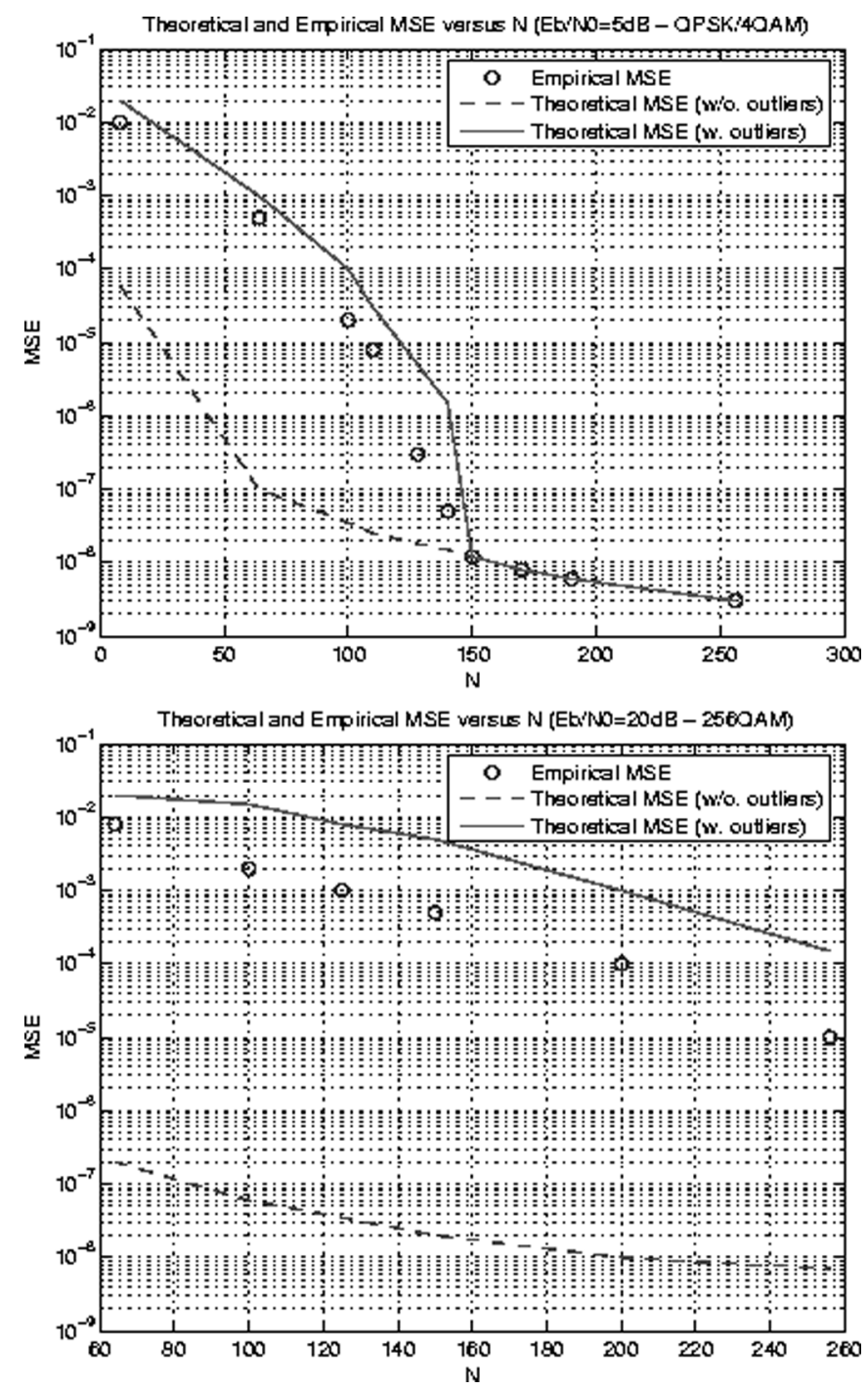

Fig. 4. Theoretical and empirical MSE versus $N$ (top: QPSK; bottom: 256QAM)

approach described in [9], which is recalled in Section I. An expression for the outlier-free MSE was derived in [4] and is given by $\mathrm{MSE}_{\text {o.f. }}=\left(3 / 32 \pi^{2}\right)\left(\left(\sigma_{e}^{2}-\Re\left\{\tilde{\sigma}_{e}^{2}\right\}\right) /|\mathcal{S}|^{2} N^{3}\right)$. We observe that the theoretical MSE is now in good agreement with the empirical MSE. According to Fig. 3, for QPSK signalling, the SNR threshold $\mathrm{SNR}_{\mathrm{th}}$ (i.e., the SNR below which the CFO estimate is grossly inaccurate) is about $6 \mathrm{~dB}$ when $N=128$. For 256 QAM and $N=128$, the outlier probability does not vanish in the absence of noise; this is due to the self-noise effect. Nevertheless, when $N$ increases, the outlier probability decreases, and thus the gap between the empirical MSE and the theoretical MSE evaluated without taking into account the outlier effect decreases (cf. Fig. 4).

\section{CONCLUSIONS}

The performance of a conventional blind CFO estimator for digital modulations was investigated in the case of low SNR/ number of samples. More specifically, we analyzed the outliers effect and derived an approximate closed-form expression for the probability of its occurrence. The closed-form expression 
was shown to be tight for most practical digital modulations. For a given SNR (or number of samples), the new results give insight into the minimum number of samples (or SNR) required in the CFO estimation procedure in order to ensure that the MSE on estimation is not significantly affected by the outliers.

\section{REFERENCES}

[1] F. Gini and G. B. Giannakis, "Frequency offset and symbol timing recovery in flat-fading channels: A cyclostationary approach," IEEE Trans. Commun., vol. 46, no. 3, pp. 400-411, Mar. 1998.

[2] A. J. Viterbi and A. M. Viterbi, "Non-linear estimation of PSK-modulated carrier phase with application to burst digital transmissions," IEEE Trans. Inf. Theory, vol. IT-29, no. 5, pp. 543-551, Jul. 1983.

[3] O. Besson and P. Stoica, "Nonlinear least-squares approach to frequency estimation and detection for sinusoidal signals with arbitrary envelope,” Digital Signal Process., vol. 9, no. 1, pp. 45-56, Jan. 1999.
[4] M. Ghogho, A. Swami, and T. Durrani, "Blind estimation of frequency offset in the presence of unknown multipath," in Proc. Int. Conf. Pers. Wireless Commun., 2000, pp. 104-108.

[5] P. Ciblat, P. Loubaton, E. Serpedin, and G. B. Giannakis, "Performance of blind carrier frequency offset estimation for non-circular transmissions through frequency-selective channels," IEEE Trans. Signal Process., vol. 50, no. 1, pp. 130-140, Jan. 2002.

[6] Y. Wang, E. Serpedin, and P. Ciblat, "Optimal blind carrier recovery for $M$-PSK burst transmissions," IEEE Trans. Commun., vol. 51, no. 9, pp. 1571-1581, Sep. 2003.

[7] - , "Optimal blind nonlinear least-squares carrier phase and frequency offset estimation for general QAM modulations," IEEE Trans. Wireless Commun., vol. 2, no. 5, pp. 1040-1054, Sep. 2003.

[8] H. Steendam and M. Moeneclaey, "Low-SNR limit of the Cramer-Rao bound for estimating the carrier phase and frequency of a PAM, PSK, or QAM waveform," IEEE Commun. Lett., vol. 5, no. 5, pp. 218-220, May 2001.

[9] D. C. Rife and R. R. Boorstyn, "Single-tone parameter estimation from discrete-time observations," IEEE Trans. Inf. Theory, vol. IT-20, no. 5, pp. 591-598, Sep. 1974.

[10] M. K. Simon and M.-S. Alouini, "A simple single integral representation of the bivariate Rayleigh distribution," IEEE Commun. Lett., vol. 2, no. 5, pp. 128-130, May 1998. 